\title{
New satellite random access preamble design based on pruned DFT-spread FBMC
}

\author{
Màrius Caus, Member, IEEE, Ana I. Pérez-Neira, Fellow Member, IEEE, Joan Bas, Senior Member, IEEE and \\ Luis Blanco, Member, IEEE,
}

\begin{abstract}
Next generation satellite payload technology is expected to provide digital processing capabilities. This will pave the way to regard satellites as flying base stations. However, the initial access procedure must be improved. In this work we focus on non-geostationary earth orbit (NGEO) satellite networks. In this scenario, the random access preamble signal and the detection must be robust to large carrier frequency offsets (CFOs). Towards this end, we investigate the adoption of the pruned discrete Fourier transform spread filter bank multicarrier waveform. The proposed design is suitable for the access scheme of forthcoming 5G-based NGEO satellite communications. The reason is twofold. First, it improves the spectral confinement with respect to the standard single-carrier frequency-division multiplexing (SC-FDM) waveform. Second, it achieves a high level of commonality with 5G new radio, by keeping unchanged the subcarrier spacing, the slot duration and the preamble sequence. Remarkably, the new design allows the straightforward application of non-coherent post detection integration (NCPDI) techniques, which divide the correlation in blocks. Numerical results show that the proposed solution reduces out-of-band emissions and the missed detection probability in presence of CFO, with respect to the conventional approach based on SCFDM and preamble detection with full-length correlation.
\end{abstract}

\section{INTRODUCTION}

W ITH the aim of providing service in remote areas and increasing service availability, it is envisioned that satellites will be an integral component of the forthcoming 5G networks. Ongoing activities and efforts are undertaken by $3 \mathrm{GPP}$ to go deeper into the role of satellites in $5 \mathrm{G}$ new radio (5G NR) [1]. Towards this end, special attention must be paid to the characteristics of the satellite environment, such as Doppler effects, large path loss and long round trip delay (RTD). In the literature, we can find several works that analyze the technical challenges associated with the convergence of satellite and terrestrial networks, [1]-[6]. To satisfy satellite communication requirements, several aspects of the terrestrial protocol need to be revisited, e.g. synchronization, random access procedures, hybrid automatic repeat request (HARQ) retransmission schemes, link adaptation and handover mechanisms, to mention a few. The purpose of this paper is to investigate the initial random access in regenerative architectures where the user equipment (UE) is served by a base station on

M. Caus, J. Bas and L. Blanco are with the Centre Tecnològic de Telecomunicacions de Catalunya (CTTC), 08860 Castelldefels, Barcelona, Spain (e-mail: \{marius.caus, joan.bas, luis.blanco\}@cttc.cat).

A.I. Pérez-Neira is with the Department of Signal Theory and Communications, Universitat Politècnica de Catalunya (UPC), 08034 Barcelona, Spain and with the Centre Tecnològic de Telecomunicacions de Catalunya (CTTC), 08860 Castelldefels, Barcelona, Spain (e-mail: \{ana.isabel.perez@upc.edu, aperez@cttc.es\}). board a satellite. In particular, we focus the attention on nongeostationary earth orbit (NGEO) satellite communications. In this scenario, if the UE knows the trajectory of the satellite and is equipped with a global navigation satellite system (GNSS) receiver, then the Doppler frequency shift can be compensated. In such a case, no obstacles are foreseen to reuse the 5G NR physical random access channel (PRACH) waveform [7]. If GNSS based techniques are not applicable at the UE side, the impairments must be handled by the network. For instance, by implementing pre-compensation techniques, it follows that any UE located in the beam center, or any other point of reference, would not experience a carrier frequency offset (CFO) due to the orbital motion. Unfortunately, if the users are located in a different spot, then they will suffer from a residual error. Depending on the altitude of the satellite and the beam size, the pre-compensation strategy may not be sufficient to cope with the Doppler effects.

Only if the differential values are tolerated by the standard, the PRACH waveform and the preamble detection can be kept unchanged with respect to the terrestrial case. Otherwise, enhancements must be made to facilitate the detection with a large CFO. In 5G NR systems, the random access signals are obtained by modulating a Zadoff-Chu (ZC) sequence onto a single-carrier frequency-division multiplexing (SC-FDM) waveform [7]. The correlation properties of these signals in presence of CFO are thoroughly analyzed in [8]-[10]. Owing to the $\mathrm{CFO}$, the correlation peak is decreased and pseudocorrelation peaks emerge, affecting the missed detection probability. To remedy this issue and improve the robustness of the initial uplink synchronization, the usage of restricted preamble sets together with peak combining is proposed in [8]. However, if the CFO is higher than the subcarrier spacing, then the correlation peak is shifted and thus, the timing is not correctly estimated. In this case, the adverse effects caused by large CFO can be more effectively mitigated by extending the detection zone, i.e. by considering multiple hypotheses of frequency offsets [11], [12]. If any of the peaks associated with a given user exceeds the detection threshold, it is considered that this user is successfully detected. Departing from the ZC sequences described in $5 \mathrm{G} \mathrm{NR}$, alternatives to the traditional method can be found in the literature. In this regard, the authors in [13], [14] have proposed a novel signal format that is based on symmetric ZC sequences. Alternatively, in [15], the random access preamble design is based on $m$-sequences. Interestingly, it was agreed in 3GPP meetings to consider the adoption of $m$-sequences to enhance the preamble [16].

In light of the above, existing solutions provide robustness 
to the CFO but do not account for the peculiarities of satellite communications. In the NGEO satellite context, the waveform for random access must have remarkable properties, such as good spectral confinement, low latency and low envelope fluctuation. Although SC-FDM presents low peak-to-average power ratio (PAPR) and good time localization properties, its frequency response decays smoothly. For this reason, we propose to adopt the pruned discrete Fourier transform spread filter bank multicarrier (DFT-s-FBMC) waveform, which does have the key features to become an eligible candidate to convey the preamble sequence [17]. A very interesting property of the solution conceived in this paper is that the preamble sequence is partitioned into blocks, which are transmitted in consecutive multicarrier symbols. At the receive side, each block is independently demodulated. Interestingly, after that, the adoption of non-coherent post detection integration (NCPDI) comes naturally [18]-[20]. The beauty of NCPDI is that it is natively more robust to CFO than conventional detectors based on the full-length correlation. As a summary the contributions of this paper are as follows:

- We have proposed a new random access preamble design, where the pruned DFT-s-FBMC scheme is used to convey the same ZC sequences adopted in 5G NR [7]. The new design improves the spectral confinement with respect to the standard solution based on SC-FDM. In addition, it achieves a high level of commonality with $5 \mathrm{G}$ NR, because the subcarrier spacing, the slot duration and the ZC sequences are kept unchanged.

- A thorough mathematical characterization has been carried out to show that the adoption of pruned DFT-s-FBMC paves the way to implementing NCPDI. A comprehensive theoretical analysis has been conducted to demonstrate that the NCPDI reduces the missed detection probability in presence of $\mathrm{CFO}$, when compared to the detector that is governed by the full-length correlation.

- Showing by simulations that the proposed preamble design together with NCPDI achieves higher robustness to CFO than the conventional approach based on SC-FDM and preamble detection with full-length correlation.

It is important to remark that NCPDI can be applied to SCFDM with similar results as pruned DFT-s-FBMC. However, by adopting SC-FDM the system is sacrificing the improved spectral confinement that is provided by pruned DFT-s-FBMC. To have all the key ingredients it is necessary to combine NCPDI and pruned DFT-s-FBMC.

The rest of the paper is organized as follows. In Section II we formulate the system model. The pruned DFT-s-FBMC scheme is then presented in Section III. Next, the new preamble design and detection method are described in Section IV and $\mathrm{V}$, respectively. The numerical results are included in Section VI and finally Section VII draws the conclusions.

Notation: Upper- and lower-case boldface letters denote matrices and vectors, respectively. Let the superscripts $(.)^{\mathrm{T}}$, $(.)^{*}$ and $(.)^{\mathrm{H}}$ denote, respectively, transpose, complex conjugate and Hermitian transpose operations. We will use $[\mathbf{A}]_{i j}$ to refer to the $(i, j)$-th element of a matrix $\mathbf{A}$. By $\mathbf{I}_{N}$ we denote the $N$ th order identity matrix. The column-reversed version of the identity matrix is denoted $\mathbf{J}_{N}$. We define $\lceil x\rceil$ and $\lfloor x\rfloor$ to be the nearest integer higher than $x$ and lower than $x$, respectively. $\operatorname{diag}(\mathbf{a})$ is a diagonal matrix with the entries of the vector a on its diagonal. We will use $\star$ to denote the convolution operation. Let $\mathbb{E}\{\cdot\}$ be the expectation operator. The symbol $\delta_{k, n}$ is 1 if $k=n$ and 0 otherwise. $\sqrt{-1}$ is denoted by $j$. The sets of $N \times M$ real- and complex-valued matrices are respectively denoted by $\mathbb{R}^{N \times M}$ and $\mathbb{C}^{N \times M}$. The upsampling and downsampling operations by a factor of $x$ will be denoted by $(.)_{\uparrow x}$ and $(.)_{\downarrow x}$, respectively.

\section{SYSTEM MODEL}

This section is devoted to characterize a multicarrier communication system that partitions the bandwidth into $M$ subchannels by employing the FBMC modulation scheme [21], [22]. In practical systems, the transmitted signal is obtained by frequency multiplexing $M_{a}$ baseband signals, where $M_{a} \leq M$. This boils down to leaving empty some subcarriers, which is mandatory to reduce out-of-band (OOB) emissions. Denoting $f_{s}$ the frequency sampling, in FBMC systems the symbols are separated $\Delta_{f}=\frac{f_{s}}{M} \mathrm{~Hz}$ and $\Delta_{t}=\frac{M}{2 f_{s}}$ seconds apart in frequency and time, respectively. Thus, the transmission of $N$ FBMC symbols can be expressed by

$$
s[n]=\sum_{k=0}^{N-1} \sum_{m=0}^{M_{a}-1} d_{m}[k] \theta_{m}[k] f_{m}\left[n-k \frac{M}{2}\right],
$$

where

$$
\begin{gathered}
f_{m}[n]=p[n] e^{j \frac{2 \pi}{M} m n} \\
\theta_{m}[k]=e^{j \frac{\pi}{2}(k+m)} .
\end{gathered}
$$

Notice that the sample index $k$ is used by low-rate signals while the high-rate signals utilize the sample index $n$. As for the notation, $d_{m}[k]$ denotes the symbol transmitted on the $m$ th subcarrier and the $k$-th time instant. The pattern created by $\theta_{m}[k]$ introduces a phase shift of $\frac{\pi}{2}$ between adjacent symbols in the time-frequency grid. The pulse shaping process depends on the real-valued and low-pass band filter $p[n]$. Without loss of generality, the system employs unitary energy pulses of length $L=K M$. Notice that the duration of the pulse is controlled by the overlapping factor $K$.

At the other end of the link, the received multicarrier signal is affected by additive white Gaussian noise (AWGN) and multipath fading. Denoting $h[n]$ the channel impulse response and $w[n]$ the noise samples, we express the received signal by $y[n]=h[n] * s[n]+w[n]$. In order to recover the information conveyed on each subcarrier, $y[n]$ is fed into a bank of filters. On the $q$-th subcarrier at $k$-th time instant, the demodulated signal is given by

$$
z_{q}[k]=\sum_{n} y[n] f_{q}^{*}\left[n-k \frac{M}{2}\right] .
$$

The demodulated signal can be compactly expressed as

$$
z_{q}[k]=\left(y[n] * f_{q}^{*}[-n]\right)_{\downarrow M / 2} .
$$

Plugging (1) into (5) yields

$$
z_{q}[k]=\sum_{m=0}^{M_{a}-1}\left(d_{m}[k] \theta_{m}[k]\right) * g_{q m}[k]+w_{q}[k],
$$


where

$$
w_{q}[k]=\left(w[n] * f_{q}^{*}[-n]\right)_{\downarrow M / 2}=\sum_{n} w[n] f_{q}^{*}\left[n-k \frac{M}{2}\right]
$$

is the filtered noise. According to the system model formulated in [22], in low frequency selective channels, the interplay between the $m$-th and the $q$-th subcarrier signal can be approximated to

$$
g_{q m}[k] \approx H_{q} \alpha_{q m}[k], \quad-L_{1} \leq k \leq L_{1},
$$

where $L_{1}=\left\lfloor\frac{L-1}{M / 2}\right\rfloor$ and

$$
\alpha_{q m}[k]=\sum_{n} p[n] p\left[n+k \frac{M}{2}\right] e^{j \frac{2 \pi}{M} n(m-q)} e^{j \pi m k} .
$$

The expression is simplified so that the equivalent channel only depends on the waveform and $H_{q}$, which represents the channel frequency response evaluated on $\omega=\frac{2 \pi}{M} q$.

Provided that the transmitted symbols are real-valued and the pulse is designed to satisfy the perfect reconstruction property, i.e.,

$$
\mathfrak{R}\left(\theta_{q}^{*}[k] \theta_{m}[k-l] \alpha_{q m}[l]\right)=\delta_{q, m} \delta_{l, 0},
$$

then the transmitted symbols can be perfectly estimated, except for the noise. In such a case, the real part should be extracted after compensating the phase term and equalizing the demodulated signals on a per-subcarrier basis, namely,

$$
\hat{d}_{q}[k]=\mathfrak{R}\left(\frac{\theta_{q}^{*}[k] z_{q}[k]}{H_{q}}\right)=d_{q}[k]+\mathfrak{R}\left(\frac{\theta_{q}^{*}[k] w_{q}[k]}{H_{q}}\right) .
$$

If the channel frequency selectivity becomes severe, then (8) is not satisfied and thus, more advanced equalization techniques must be considered [22].

\section{A. Matrix notation}

To ease the analytical tractability of the signal and benefit from the matrix algebra it is of paramount importance to formulate the system model using a matrix notation. To this end, we stack column-wise the demodulated signals as

$$
\begin{gathered}
\mathbf{y}[k]=\boldsymbol{\Theta}^{\mathrm{H}}[k]\left[z_{0}[k], \cdots, z_{M_{a}-1}[k]\right]^{\mathrm{T}}= \\
\sum_{l=-L_{1}}^{L_{1}} \mathbf{H} \Theta^{\mathrm{H}}[k] \mathbf{G}[l] \Theta[k-l] \mathbf{d}[k-l]+\boldsymbol{\Theta}^{\mathrm{H}}[k] \mathbf{w}[k] .
\end{gathered}
$$

To get (12) we have defined $\mathbf{H}=\operatorname{diag}\left(\left[H_{0}, \cdots, H_{M_{a}-1}\right]\right)$ and $\boldsymbol{\Theta}[k]=\operatorname{diag}\left(\left[\theta_{0}[k], \cdots, \theta_{M_{a}-1}[k]\right]\right)$ and the vectors $\mathbf{d}[k]=$ $\left[d_{0}[k], \cdots, d_{M_{a}-1}[k]\right]^{\mathrm{T}}$ and $\mathbf{w}[k]=\left[w_{0}[k] \cdots w_{M_{a}-1}[k]\right]^{\mathrm{T}}$. The coupling between the $q$-th and the $m$-th subcarriers is given by $[\mathbf{G}[l]]_{q m}=\alpha_{q m}[l]$, for $q, m=0, \cdots, M_{a}-1$. From (9) it can be inferred that $\alpha_{q m}[l]=\alpha_{(q-m) 0}[l] e^{j \pi m l}$. Therefore, $\mathbf{G}[l]$ can be factorized as the product of a Toeplitz matrix by $\boldsymbol{\beta}_{l}=\operatorname{diag}\left(\left[e^{j \pi 0 l}, \cdots, e^{j \pi\left(M_{a}-1\right) l}\right]\right)$, namely,

$$
\mathbf{G}[l]=\left[\begin{array}{cccc}
\alpha_{00}[l] & \alpha_{(-1) 0}[l] & \cdots & \alpha_{\left(-M_{a}+1\right) 0}[l] \\
\vdots & \vdots & \ddots & \vdots \\
\alpha_{\left(M_{a}-2\right) 0}[l] & \alpha_{\left(M_{a}-3\right) 0}[l] & \cdots & \alpha_{(-1) 0}[l] \\
\alpha_{\left(M_{a}-1\right) 0}[l] & \alpha_{\left(M_{a}-2\right) 0}[l] & \cdots & \alpha_{00}[l]
\end{array}\right]
$$

According to (7), the covariance matrix of $\mathbf{w}[k]$ is given by

$$
\mathbb{E}\left\{\mathbf{w}[k] \mathbf{w}^{\mathrm{H}}[k]\right\}=N_{0} \mathbf{G}[0],
$$

provided that the noise samples at the input of the receiver are zero-mean circularly symmetric complex Gaussian random variables, i.e. $w[n] \sim C \mathcal{N}\left(0, N_{0}\right)$.

Although the multipath fading impact will not be evaluated in this work, the system model formulated in (12) is as generic as possible, as it includes a frequency selective channel.

Now imagine that we specify the matrix format (13) for the case where $M_{a}=M$. When this property is satisfied, every column of the Toeplitz matrix is computed by circularly shifting the column vector

$$
\alpha[l]=\left[\alpha_{00}[l], \alpha_{10}[l], \cdots, \alpha_{\left(M_{a}-1\right) 0}[l]\right]^{\mathrm{T}} .
$$

Matrices of this form, which are widely studied in the literature, lie within the category of the circulant matrices. For $M_{a}=M, \mathbf{G}[l]$ can be expressed as function of the DFT matrix $\mathbf{F}_{M_{a}}=\left[\omega_{0}, \cdots, \omega_{M_{a}-1}\right]^{\mathrm{T}}$, yielding

$\mathbf{G}^{c}[l]=\mathbf{F}_{M_{a}}^{\mathrm{H}} \boldsymbol{\Lambda}_{l} \mathbf{F}_{M_{a}} \boldsymbol{\beta}_{l}=\mathbf{F}_{M_{a}}^{\mathrm{H}} \operatorname{diag}\left(\left[\lambda_{0}^{l}, \cdots, \lambda_{M_{a}-1}^{l}\right]\right) \mathbf{F}_{M_{a}} \boldsymbol{\beta}_{l}$,

where $\omega_{m}=\frac{1}{\sqrt{M_{a}}}\left[e^{-j \frac{2 \pi}{M_{a}} m 0}, \cdots, e^{-j \frac{2 \pi}{M_{a}} m\left(M_{a}-1\right)}\right]^{\mathrm{T}}$ and

$$
\lambda_{m}^{l}=\sum_{q=0}^{M_{a}-1} \alpha_{q 0}[l] e^{-j \frac{2 \pi}{M_{a}} q m},
$$

for $m=0, \cdots, M_{a}-1$. The main characteristic of FBMC systems is that only a reduced number of subcarriers contribute to inter-carrier interference. Usually the transmultiplexer response is such that $\alpha_{q 0}[l] \approx 0$, for $|q|>5$. Focusing the attention to this case, it appears that the actual matrix $\mathbf{G}[l]$ and (16) differ in very few entries. Relying on this feature we assume that $\mathbf{G}[l]$ is asymptotically equivalent to (16). In conclusion, if $M_{a}$ if sufficiently large, we can benefit from the unique properties of the circulant matrices, without incurring in a significant error.

In the following, we thoroughly analyze the structure of the transmultiplexer response when $L=M$ and $M_{a}=M$, which allows us to replace $\mathbf{G}[0]$ by $\mathbf{G}^{c}[0]$. The most relevant results can be summarized in two propositions.

Proposition 1: If the prototype pulse has a duration of $L=$ $M$ samples and satisfies the perfect reconstruction property formulated in (10), then the column vector $\boldsymbol{\alpha}_{0}$ is real-valued.

Proposition 2: If $M_{a}=M$ and the elements of $\alpha_{0}$ are realvalued, then it follows that $\Lambda_{0} \in \mathbb{R}^{M_{a} \times M_{a}}$.

The details that prove mathematically Propositions 1 and 2 are provided in the Appendix A and the Appendix B, respectively. Leveraging on Propositions 1 and 2, we will show in the following section that under some conditions it is possible to project the desired data and the interference onto orthogonal subspaces.

\section{PRuned DFT-SPREAd FBMC}

This section introduces the pruned DFT-s-FBMC scheme [17]. In the rest of the section, unless otherwise stated, it will be assumed that $M_{a}$ and $M$ are chosen such that the 


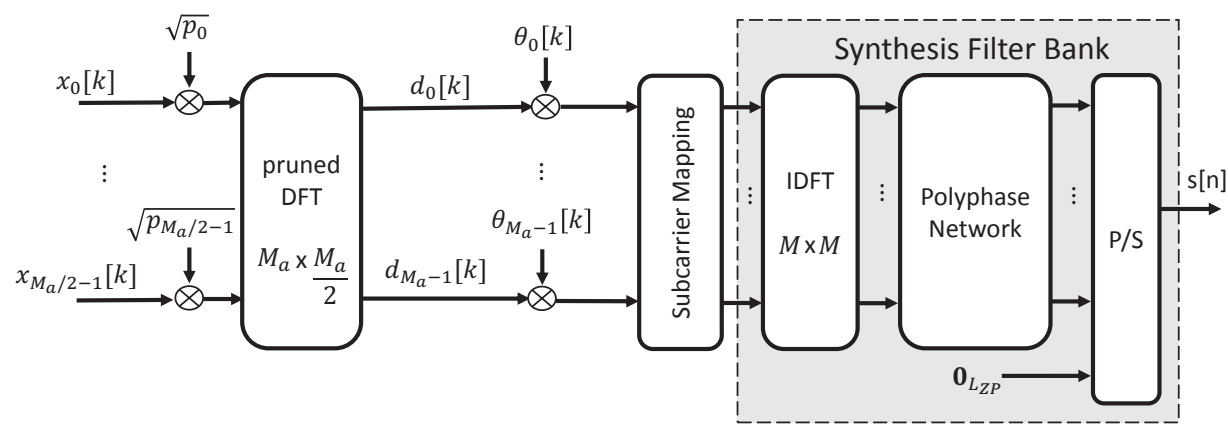

Fig. 1. Transmitter block diagram of a pruned DFT spread FBMC scheme.

residual error of assuming that $\mathbf{G}[l]$ is equivalent to (16) is negligible. Now suppose that $M_{a} / 2$ symbols are spread over the subcarriers by precoding the vector of data symbols $\mathbf{x}[k] \in \mathbb{C}^{M_{a} / 2 \times 1}$ as

$$
\mathbf{d}[k]=\mathbf{C P x}[k] .
$$

The precoding matrix is a combination of a pruned DFT matrix $\mathbf{C} \in \mathbb{C}^{M_{a} \times M_{a} / 2}$ and a diagonal matrix $\mathbf{P}=$ $\operatorname{diag}\left(\left[\sqrt{P_{0}}, \cdots, \sqrt{P_{M_{a} / 2-1}}\right]\right)$ that weights the symbols with the power coefficients. By setting $L=M$ and choosing the column vectors of $\mathbf{C}$ as

$$
\mathbf{C}=\left[\omega_{M_{a}-1}, \cdots, \omega_{M_{a} / 2}\right],
$$

we will show that under some conditions and for $l=-1,1$,

$$
\begin{gathered}
\mathbf{C}^{\mathrm{H}} \boldsymbol{\Theta}^{\mathrm{H}}[k] \mathbf{G}^{c}[l] \boldsymbol{\Theta}[k-l] \mathbf{C}= \\
\mathbf{C}^{\mathrm{H}} \boldsymbol{\Theta}^{\mathrm{H}}[k] \mathbf{F}_{M_{a}}^{\mathrm{H}} \boldsymbol{\Lambda}_{l} \mathbf{F}_{M_{a}} \boldsymbol{\beta}_{l} \boldsymbol{\Theta}[k-l] \mathbf{C}=\mathbf{0}_{\frac{M_{a}}{2} \times \frac{M_{a}}{2}} \\
\mathbf{C}^{\mathrm{H}} \boldsymbol{\Theta}^{\mathrm{H}}[k] \mathbf{G}^{c}[0] \boldsymbol{\Theta}[k] \mathbf{C}=\mathbf{C}^{\mathrm{H}} \boldsymbol{\Theta}^{\mathrm{H}}[k] \mathbf{F}_{M_{a}}^{\mathrm{H}} \boldsymbol{\Lambda}_{0} \mathbf{F}_{M_{a}} \boldsymbol{\Theta}[k] \mathbf{C}=\mathbf{D},
\end{gathered}
$$

where $\mathbf{D} \in \mathbb{C}^{M_{a} / 2 \times M_{a} / 2}$ is some diagonal matrix. We define by $\mathbf{0}_{M \times N}$ an $M \times N$ all-zeroes matrix. Concerning (20), notice that $\boldsymbol{\Theta}[k], \boldsymbol{\Theta}[k-l]$ and $\boldsymbol{\beta}_{l}$ can be regarded as row permutation matrices when they are multiplied by the DFT matrix, i.e.,

$$
\begin{gathered}
\mathbf{F}_{M_{a}} \boldsymbol{\Theta}[k]=e^{j \frac{\pi}{2} k}\left[\omega_{M_{a} / 4}, \cdots, \omega_{0}, \omega_{M_{a}-1}, \cdots, \omega_{M_{a} / 4+1}\right]^{\mathrm{H}} \\
\mathbf{F}_{M_{a}} \boldsymbol{\beta}_{l} \Theta[k-l]=e^{j \frac{\pi}{2}(k-l)} \times \\
{\left[\omega_{3 M_{a} / 4}, \cdots, \omega_{0}, \omega_{M_{a}-1}, \cdots, \omega_{3 M_{a} / 4+1}\right]^{\mathrm{H}} .}
\end{gathered}
$$

Upon projecting (22) and (23) onto $\mathbf{C}$, we get two matrices that span orthogonal subspaces. Consequently, (20) is satisfied. To prove the diagonal structure, it just needs to plug (19) and (22) into (21).

An immediate result of (20) and (21) is that the receiver can get rid of the interference, by filtering the demodulated signal $\mathbf{y}[k]$ by the decoding matrix $\mathbf{A}^{\mathrm{H}}=\mathbf{P C}^{\mathrm{H}} \mathbf{H}^{-1} \in \mathbb{C}^{M_{a} / 2 \times M_{a}}$. Furthermore, if $\mathbf{P}$ is defined by

$$
\mathbf{P}=\operatorname{diag}\left(\left[\frac{1}{\sqrt{\lambda_{M_{a} / 4+1}^{0}}}, \cdots, \frac{1}{\sqrt{\lambda_{3 M_{a} / 4}^{0}}}\right]\right),
$$

it follows that $\mathbf{P C}^{\mathrm{H}} \mathbf{\Theta}^{\mathrm{H}}[k] \mathbf{G}^{c}[0] \mathbf{\Theta}[k] \mathbf{C P}=\mathbf{I}_{M_{a} / 2}$.

Proposition 3: If Proposition 2 remains true and $\left|\alpha_{10}[0]\right|>>$ $\left|\alpha_{q 0}[0]\right|$, for $q>1$, then the $M_{a} / 2$ dominant eigenvalues of
$\mathbf{G}^{c}[0]$ are given by $\left\{\lambda_{M_{a} / 4+1}^{0}, \cdots, \lambda_{3 M_{a} / 4}^{0}\right\}$, as long as $\alpha_{10}[0]<$ 0 .

The proof is provided in Appendix C. Taking into account Proposition 3 and the permutation induced by $\boldsymbol{\Theta}[k]$, which is formulated in (22), it can be readily checked that the eigenvectors associated with the highest eigenvalues are activated only if the precoder is equal to (19).

By allocating the power inversely proportional to the magnitude of the eigenvalues, the equalized signal can be compactly expressed as

$$
\hat{\mathbf{x}}[k]=\mathbf{A}^{\mathrm{H}} \mathbf{y}[k]=\mathbf{x}[k]+\mathbf{P C}^{\mathrm{H}} \mathbf{H}^{-1} \boldsymbol{\Theta}^{\mathrm{H}}[k] \mathbf{w}[k] .
$$

This result analytically proves that the symbols can be received in the absence of interference if $L=M$ and the number of active carriers, which is determined by $M_{a}$, is sufficiently large. As the authors emphasize in [17], the pruned DFT-sFBMC scheme does not sacrifice the rate, provided that the components of the data symbol vector $\mathbf{x}[k]$ are drawn from a QAM constellation. Building upon the results presented in this section, next we will tackle the random access preamble design.

\section{Preamble Design}

The basis of the PRACH in 3GPP technical specifications [7] is formed by the class of $\mathrm{ZC}$ sequences

$$
x_{u}(i)=e^{-j \frac{\pi u i(i+1)}{N_{\mathrm{ZC}}}},
$$

where $i=0, \cdots, N_{\mathrm{ZC}}-1$ and $u=1, \cdots, N_{\mathrm{ZC}}-1$. The term $N_{\mathrm{ZC}}$ represents the period of the sequence. For a fixed root $u$, the preambles are generated by cyclically shifting $x_{u}(i)$, i.e.,

$$
x_{u, v}(i)=x_{u}\left(\left(i+C_{v}\right) \bmod N_{\mathrm{ZC}}\right) \propto x_{u}(i) e^{-j \frac{2 \pi}{N_{\mathrm{ZC}}} u C_{v} i} .
$$

The shift $C_{v}$ is picked from this set $\left\{0,1, \cdots, N_{\mathrm{ZC}}-1\right\}$. The preambles that are generated from the same $\mathrm{ZC}$ sequence are orthogonal as long as the cyclic shift is different. Conversely, the preambles that are obtained by cyclically shifting ZC sequences with different root values are not orthogonal. In this case, the correlation between $x_{u_{1}, v_{1}}(i)$ and $x_{u_{2}, v_{2}}(i)$ is $\frac{1}{\sqrt{N_{\mathrm{ZC}}}}$, if $N_{\mathrm{ZC}}$ is a prime number and $u_{1} \neq u_{2}$.

Once the terminal picks one preamble from the sequences available, $x_{u, v}(i)$ is mapped into the time-frequency resources of the random access channel. To this end, the pruned DFT-sFBMC modulation scheme will be considered. The efficient 


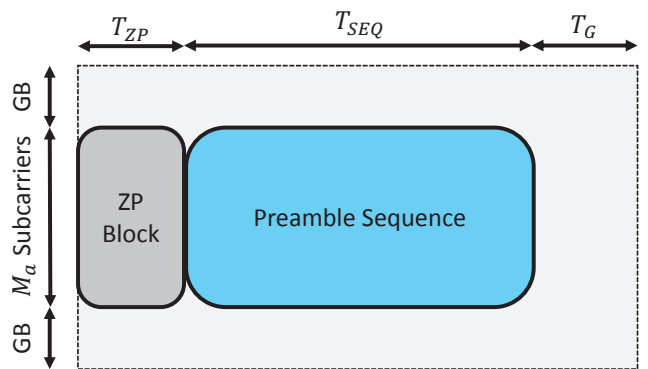

Fig. 2. Structure of the random access channel.

implementation of the transmitter that is proposed in [21] is depicted in Figure 1. The polyphase network becomes an element-wise multiplication with the prototype pulse when $L=M$. Unlike standard SC-FDM systems, circularity is not required in pruned DFT-s-FBMC and thus, the cyclic prefix (CP) can be replaced by a vector of $L_{Z P}$ zeroes that is transmitted ahead of the sequence. It is clear that the zero padding (ZP) block should be dimensioned according to the maximum delay spread. Following the ZP approach, robustness is provided against the leakage of previous frames. In addition, a variable guard time (GT) is added at the end of the preamble to take into account the propagation delays. The proposed structure is depicted in Figure 2. By $T_{\mathrm{ZP}}, T_{\mathrm{SEQ}}$ and $T_{\mathrm{G}}$, we denote the duration of the ZP block, the preamble sequence and the GT, respectively. It should be underlined that part of the spectrum remains unused by placing a guard band (GB) on both sides.

Starting from (18), we assume that the preamble sequence is mapped into $M_{a}$ subcarriers and $N$ multicarrier symbols. To ensure that the preamble fits into the physical resources, we set $N=\left\lceil\frac{2 N_{\mathrm{ZC}}}{M_{a}}\right\rceil$. This translates into creating a zero padded preamble sequence that reads as

$$
\bar{x}_{u, v}(i)=\left\{\begin{array}{cl}
x_{u, v}(i) & 0 \leq i \leq N_{\mathrm{ZC}}-1 \\
0 & N_{\mathrm{ZC}} \leq i \leq N \frac{M_{a}}{2}-1 .
\end{array}\right.
$$

Then, the elements of the vector to be fed into the precoder are defined as $[\mathbf{x}[k]]_{m}=\bar{x}_{u, v}\left(k M_{a} / 2+m\right)$, for $k=0, \cdots, N-1$ and $m=0, \cdots, M_{a} / 2-1$. Since $\mathbf{F}_{M_{a}}$ is a symmetric unitary matrix, the precoded symbol vector can be expressed as follows:

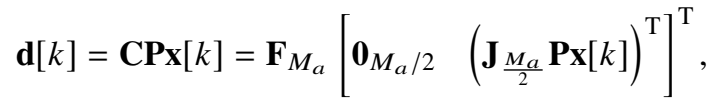

where $\mathbf{0}_{x}$ is a row vector of zeros having a length $x$. Figure 1 reveals that the precoded symbols at the input of the $M \times M$ IDFT block are multiplied by $\theta_{m}[k]$. This implies that the precoded symbol vector at the input of the synthesis filter bank becomes

$$
\boldsymbol{\Theta}[k] \mathbf{d}[k]=e^{j \frac{\pi}{2} k} \mathbf{F}_{M_{a}}\left[\begin{array}{lll}
\mathbf{0}_{M_{a} / 4} & \left(\mathbf{J}_{\frac{M_{a}}{2}} \mathbf{P x}[k]\right)^{\mathrm{T}} & \mathbf{0}_{M_{a} / 4}
\end{array}\right]^{\mathrm{T}} .
$$

From the theory developed in [17], [23], we can conclude that upon mapping (30) onto the corresponding subcarriers and executing the IDFT processing, most of the energy of the $k$-th multicarrier symbol comes from the samples that are indexed

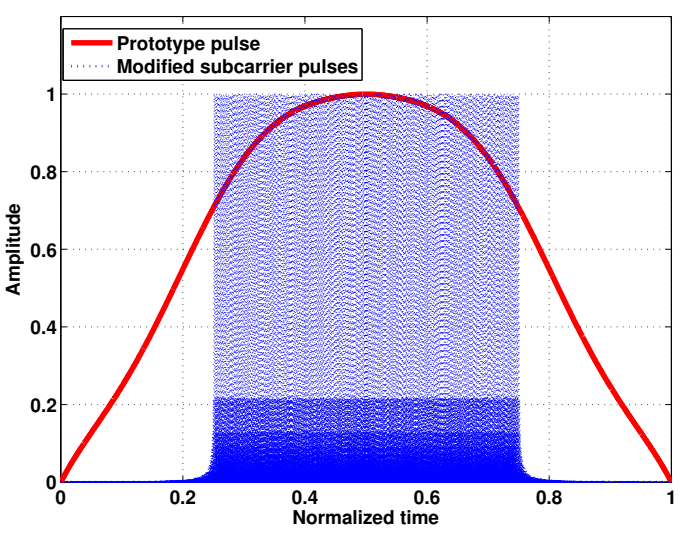

Fig. 3. Time response of the modified subcarrier pulses and the prototype pulse for $M=6144$ and $M_{a}=840$.

by $n=\frac{M}{4}+k \frac{M}{2}, \cdots, \frac{3 M}{4}+k \frac{M}{2}$. To show this result, it is helpful to express the $k$-th multicarrier symbol

$$
s_{k}[n]=\sum_{m=0}^{M_{a}-1} f_{m}\left[n-k \frac{M}{2}\right] \theta_{m}[k] d_{m}[k],
$$

as a time domain vector $\mathbf{s}_{k}=$ $\left[s_{k}[k M / 2], \cdots, s_{k}[k M / 2+L-1]\right]^{\mathrm{T}}$ that depends on the subcarrier pulse vectors $\mathbf{f}_{m}=\left[f_{m}[0], \cdots, f_{m}[L-1]\right]^{\mathrm{T}}$ and the precoded symbol vector as follows:

$$
\mathbf{s}_{k}=\left[\mathbf{f}_{0}, \cdots, \mathbf{f}_{M_{a}-1}\right] \boldsymbol{\Theta}[k] \mathbf{C P x}[k]=\left[\hat{\mathbf{f}}_{0}, \cdots, \hat{\mathbf{f}}_{M_{a} / 2-1}\right] \mathbf{x}[k] .
$$

It can be noticed that $\boldsymbol{\Theta}[k] \mathbf{C P} \in \mathbb{C}^{M_{a} \times M_{a} / 2}$ transforms the original $M_{a}$ subcarrier pulses into a new basis that is formed by $M_{a} / 2$ pulses. In Figure 3 we represent the time response of $\left\{\hat{\mathbf{f}}_{0}, \cdots, \hat{\mathbf{f}}_{M_{a} / 2-1}\right\}$ and the prototype pulse, which corresponds to the time-frequency localization design [24]. As Figure 3 illustrates, the new basis improves the time localization of the multicarrier symbols. Based on this we can resolve that the the energy of the samples located at the tail and the head of the preamble is considerably lower than the average power. Leveraging on this property we improve both the protection of already uplink synchronized UEs and the resilience to the interference that comes from previous subframes.

At the receive side, the scheme of the transmitter is reversed. A schematic view of the receiver is shown in Figure 4. First of all the samples of the ZP block are discarded. Then, the signal is fed into a bank of filters and the output is converted to the frequency domain. Next, the used subcarriers are retrieved from the output of the DFT block, the phase is compensated and finally the decoding matrix $\mathbf{P C}^{\mathrm{H}} \in \mathbb{C}^{M_{a} / 2 \times M_{a}}$ is used to equalize the signal. Building upon the proposed transceiver, Section $\mathrm{V}$ tackles the detection algorithm.

\section{Preamble detection}

To determine if any user is requesting access in the physical resources of the random access channel, the base station uses a sliding window with the same duration as the preamble sequence. In each window, the detector searches if there is 


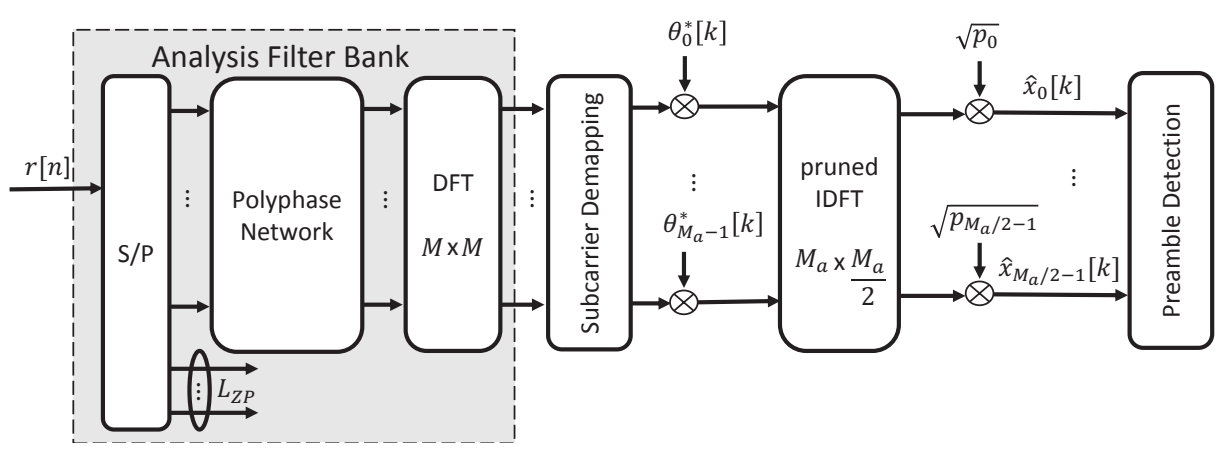

Fig. 4. Receiver block diagram of a pruned DFT spread FBMC scheme.

any signature that matches with the local sequences. If so, information related to the time lag is passed to the time advance module. The first window starts at $T_{\mathrm{ZP}}$ to remove the ZP block, while successive windows are shifted to cover all possible delays owing to the user position uncertainty. Consecutive windows are shifted a multiple of the sampling period $T_{s}$, which depends on the desired time granularity. The set of possible shifts is determined by the search window size $D_{w}$, which in turn, is related to the cell size. The application of the sliding window implies executing multiple times the analysis filter bank (AFB). The efficient implementation of the receiver, where the AFB is executed once and all the processing is done at the output of the DFT block, is left for future work.

To ease the understanding of the detection method, first of all we will assume an ideal situation, where the channel does not induce any distortion and the delay of the window perfectly matches with the RTD. This is equivalent to particularize (25) to $h[n]=\delta[n]$. Then, the signal at the input of the detector can be compactly formulated as

$$
\hat{\mathbf{x}}[k]=\mathbf{x}[k]+\mathbf{P C}^{\mathrm{H}} \boldsymbol{\Theta}^{\mathrm{H}}[k] \mathbf{w}[k] .
$$

Making use of $\hat{\mathbf{x}}[0], \cdots, \hat{\mathbf{x}}[N-1]$, the preamble detection relies on the NCPDI method [18]-[20]. Unlike the conventional approach, the proposed method divides the demodulated data into $N$ blocks and then computes the correlation with the candidate preamble sequences on a block basis as follows:

$$
\rho_{v v}=\sum_{k=0}^{N-1} \frac{1}{\left|N_{\mathrm{ZC}}\right|^{2}}\left|\rho_{v v}^{k}\right|^{2},
$$

where

$$
\rho_{v v}^{k}=\sum_{m=0}^{\frac{M_{a}}{2}-1}[\hat{\mathbf{x}}[k]]_{m} \bar{x}_{v, v}^{*}\left(k \frac{M_{a}}{2}+m\right) .
$$

It is noteworthy to mention that the reference preamble sequence is characterized by the tuple $\left(v, C_{v}\right)$, which respectively denotes the root index and the cyclic shift. The use of the NCPDI method is endorsed by the increased robustness against the phase uncertainty that stems from the CFO. We have favored NCDPI over other PDI methods because it can be analytically characterized, which is helpful to formulate the detector threshold in a closed-form expression. Supposing that the transmitted preamble is represented by $\left(u, C_{\mu}\right)$, then it follows that

$$
\rho_{\nu v}^{k} \approx \begin{cases}e^{j \phi(u, v, \mu)} \frac{\sin \left(\frac{\pi u\left(C_{\mu}-C_{v}\right) M_{a}}{\left.2 N_{\mathrm{ZC}}\right)}\right)}{\sin \left(\frac{\pi u\left(C_{\mu}-C_{v}\right)}{N_{\mathrm{ZC}}}\right)}+\eta_{u v}^{k} & u=v \\ \eta_{v v}^{k} & u \neq v,\end{cases}
$$

where $\eta_{\nu v}^{k}$ is the additive noise that degrades the correlation and $\phi(u, v, \mu)$ is a phase term that depends on the transmitted and the reference preamble sequences. Although orthogonality is not satisfied when $u \neq v$, we will assume that the residual error is negligible in the signal-to-noise ratio (SNR) range of interest.

At this point, it is worth recalling that Section III has shown that in the asymptotic regime the equivalent channel exhibits a diagonal structure, i.e., $\mathbf{P C}^{\mathrm{H}} \boldsymbol{\Theta}^{\mathrm{H}}[k] \mathbf{G}^{c}[0] \boldsymbol{\Theta}[k] \mathbf{C P}=$ $\mathbf{I}_{M_{a} / 2}$. Bearing this result in mind together with the statistical information provided in (14), we can claim that the noise samples at the input of the detector are independent and identically distributed with mean 0 and variance $N_{0}$. Therefore, the correlation noise within $\rho_{\nu v}^{k}$ obeys this distribution $\eta_{\nu v}^{k} \sim$ $C \mathcal{N}\left(0, N_{0} M_{a} / 2\right)$. As a consequence, the noise component of $\rho_{v v}$ has a chi-square distribution with $2 N$ degrees of freedom [20]. This statistical information is harnessed to derive the detection threshold $r_{\text {th }}$ in terms of the false alarm probability. In this sense, when no preamble is transmitted, the probability that $\rho_{\nu v}$ is above $r_{\text {th }}$ is given by

$$
P_{\mathrm{FA}}=1-\left(1-e^{-\frac{r_{\mathrm{t}}\left|N_{\mathrm{ZC}}\right|^{2}}{N_{0} M a / 2}} \sum_{k=0}^{N-1} \frac{1}{N !}\left(\frac{r_{\mathrm{th}}\left|N_{\mathrm{ZC}}\right|^{2}}{N_{0} M_{a} / 2}\right)^{k}\right)^{D_{w}},
$$

where $D_{w}$ is the window search size [25].

\section{A. Impact of TO and CFO on the detection}

This section is devoted to characterize the impact of the time offset (TO) and the CFO on the NCPDI method. Due to its analytical tractability we focus on the scenario where the preamble is detected over a line-of-sight (LoS) channel with $L=M$. Under these assumptions, the received signal is given by $y[n]=s[n-\tau] e^{j \frac{2 \pi}{M} \epsilon n}+w[n]$, where $s[n]$ is the random access signal, $w[n]$ is the additive noise, $\tau \in \mathbb{Z}$ is the TO normalized to the sampling period and $\epsilon \in \mathbb{R}$ is the CFO normalized to the subcarrier spacing. In order to concentrate on the detrimental effects induced by the TO and the CFO we neglect $w[n]$. Since the noise is uncorrelated with the data, 
we do not envisage any effect on the analysis owing to the simplification. Now, (6) becomes

$$
z_{q}[k]=e^{j \pi \epsilon k} \sum_{m=0}^{M_{a}-1}\left(d_{m}[k] \theta_{m}[k]\right) * g_{q m}^{\tau \epsilon}[k]
$$

where

$$
\begin{aligned}
g_{q m}^{\tau \epsilon}[k]= & \left(\sum_{n} e^{j \frac{2 \pi}{M} \epsilon n} p[n] p\left[n-\tau+k \frac{M}{2}\right] e^{j \frac{2 \pi}{M} n(m-q)}\right) \\
& \times e^{j \pi m k} e^{-j \frac{2 \pi}{M} m \tau} .
\end{aligned}
$$

Analogously to the procedure followed in Section II-A, the communication model can be formulated as

$$
\mathbf{y}[k]=\sum_{l} e^{j \pi \epsilon k} \boldsymbol{\Theta}^{\mathrm{H}}[k] \mathbf{G}_{\tau \epsilon}[l] \boldsymbol{\Theta}[k-l] \mathbf{d}[k-l] .
$$

As evidenced from (39), the response can be expressed as $g_{q m}^{\tau \epsilon}[k]=g_{(q-m) 0}^{\tau \epsilon}[k] e^{j \pi m k} e^{-j \frac{2 \pi}{M} m \tau}$. If $M_{a}$ is sufficiently large, we can invoke the circulant matrix approximation. As a result,

$$
\begin{aligned}
\mathbf{G}_{\tau \epsilon}[l]= & {\left[\begin{array}{cccc}
g_{00}^{\tau \epsilon}[l] & g_{(-1) 0}^{\tau \epsilon}[l] & \cdots & g_{\left(-M_{a}+1\right) 0}^{\tau \epsilon}[l] \\
\vdots & \vdots & \ddots & \vdots \\
g_{\left(M_{a}-2\right) 0}^{\tau \epsilon}[l] & g_{\left(M_{a}-3\right) 0}^{\tau \epsilon}[l] & \cdots & g_{(-1) 0}^{\tau \epsilon}[l] \\
g_{\left(M_{a}-1\right) 0}^{\tau \epsilon}[l] & g_{\left(M_{a}-2\right) 0}^{\tau \epsilon}[l] & \cdots & g_{00}^{\tau \epsilon}[l]
\end{array}\right] } \\
\times \boldsymbol{\beta}_{l}^{\tau}, &
\end{aligned}
$$

tends to $\mathbf{G}_{\tau \epsilon}^{c}[l]=\mathbf{F}_{M_{a}}^{\mathrm{H}} \boldsymbol{\Xi}_{l} \mathbf{F}_{M_{a}} \boldsymbol{\beta}_{l}^{\tau}$. Using the same format as (16), we build the matrices $\boldsymbol{\beta}_{l}^{\tau}=\operatorname{diag}\left(\left[e^{j \frac{2 \pi}{M} 0\left(l \frac{M}{2}-\tau\right)}, \cdots, e^{j \frac{2 \pi}{M}\left(M_{a}-1\right)\left(l \frac{M}{2}-\tau\right)}\right]\right)$ and $\boldsymbol{\Xi}_{l}=\operatorname{diag}\left(\left[\xi_{0}^{l}, \cdots, \xi_{M_{a}-1}^{l}\right]\right)$.

Proposition 4: If $\mathbf{G}_{\tau \epsilon}[l]$ reduces to $\mathbf{G}_{\tau \epsilon}^{c}[l]$, then the eigenvalues gathered in the main diagonal of $\boldsymbol{\Xi}_{l}$ can be approximated to

$$
\begin{gathered}
\xi_{m}^{l} \approx e^{-j \frac{2 \pi}{M_{a}} m \epsilon} \bar{\xi}_{m}^{l}=e^{-j \frac{2 \pi}{M_{a}} m \epsilon} e^{j 2 \pi \epsilon} M_{a} \\
\times p\left[M-m \frac{M}{M_{a}}\right] p\left[M-m \frac{M}{M_{a}}-\tau+l \frac{M}{2}\right] .
\end{gathered}
$$

The assumptions that have been made to derive (42) are provided in Appendix D. From here onwards, it will be assumed that the mismatch between $\mathbf{G}_{\tau \epsilon}[l]$ and $\mathbf{G}_{\tau \epsilon}^{c}[l]$ leads to a negligible modeling error. Accordingly, after applying the transmit and the receive processing described in Section III, the equalized signal vector is formulated as $\hat{\mathbf{x}}[k]=\mathbf{P C}^{\mathrm{H}} \mathbf{y}[k]$. At the input of the detector, the channel seen by $\mathbf{x}[k-l]$ is given by

$$
\mathbf{H}_{\mathrm{T}}[l]=\mathbf{P C}^{\mathrm{H}} \boldsymbol{\Theta}^{\mathrm{H}}[k] \mathbf{F}_{M_{a}}^{\mathrm{H}} \boldsymbol{\Xi}_{l} \mathbf{F}_{M_{a}} \boldsymbol{\beta}_{l}^{\tau} \boldsymbol{\Theta}[k-l] \mathbf{C P} .
$$

It is important to remark that the time misalignment leads to a phase shift that translates into a row permutation of the DFT matrix. In light of this observation, (23) can be generalized as

$$
\begin{aligned}
\mathbf{F}_{M_{a}} \boldsymbol{\beta}_{l}^{\tau} \Theta[k-l]= & e^{j \frac{\pi}{2}(k-l)} \times \\
& {\left[\omega_{b}, \cdots, \omega_{0}, \omega_{M_{a}-1}, \cdots, \omega_{b+1}\right]^{\mathrm{H}}, }
\end{aligned}
$$

where $b=M_{a} \frac{3}{4}-\tau \frac{M_{a}}{M}$ and $l=-1,1$. For the ease of exposition we have considered the case where $b$ is an integer number.
After carefully analyzing (19), (22), (42), (43) and (44), it can be verified that after several derivation steps we get

$$
\begin{aligned}
& \mathbf{H}_{\mathrm{T}}[0] \approx \operatorname{diag}\left(\left[\frac{\bar{\xi}_{\frac{M_{a}}{4}+1}^{0}}{\sqrt{\frac{\lambda_{\frac{M_{a}}{4}+1+\tau} M_{a}}{\lambda^{0}}{ }^{0} \frac{M_{a}+1}{4}}}, \cdots,\right.\right.
\end{aligned}
$$

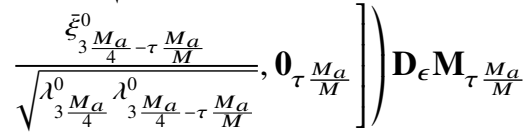

$$
\begin{aligned}
& \mathbf{H}_{\mathrm{T}}[l] \approx j^{-l} \operatorname{diag}\left(\left[\mathbf{0}_{\frac{M_{a}}{2}-\tau \frac{M_{a}}{M}}, \frac{\bar{\xi}_{3 \frac{M_{a}}{4}-\tau \frac{M_{a}}{M}+1}}{\sqrt{\lambda_{\frac{M_{a}}{4}+1}^{\lambda_{3}^{0}} \frac{M_{a}}{4}-\tau \frac{M_{a}}{M}+1}},\right.\right. \\
& \left.\left.\cdots, \frac{\bar{\xi}_{3 \frac{M_{a}}{4}}^{l}}{\sqrt{\frac{\lambda_{\frac{M_{a}}{4}+\tau \frac{M_{a}}{M}}^{\lambda^{0}}{ }_{3 \frac{M_{a}}{4}}}{n}}}\right]\right) \mathbf{D}_{\epsilon} \mathbf{M}_{\tau \frac{M_{a}}{M}}
\end{aligned}
$$

where $l=-1,1$ and

$$
\begin{gathered}
\mathbf{M}_{L}=\left[\begin{array}{cc}
\mathbf{0}_{\frac{M_{a}}{2}-L \times L} & \mathbf{I}_{\frac{M_{a}}{2}-L} \\
\mathbf{I}_{L} & \mathbf{0}_{L \times \frac{M_{a}}{2}-L}
\end{array}\right] \\
\mathbf{D}_{\epsilon}=\operatorname{diag}\left(\left[e^{-j \frac{2 \pi}{M_{a}} \epsilon\left(\frac{M_{a}}{4}+1\right)}, \cdots, e^{-j \frac{2 \pi}{M_{a}} \epsilon\left(3 \frac{M_{a}}{4}\right)}\right]\right) .
\end{gathered}
$$

If we focus on the effect of the TO, it is clear from (47) that the cyclic permutation matrix transforms the $k$-th block into $\mathbf{M}_{\tau \frac{M_{a}}{M}} \mathbf{x}[k]$. Provided that the blocks are cyclically shifted $\tau \frac{M_{a}}{M}$ positions, the detection function formulated in (34) will produce a peak when the transmitted and the local preambles are both $\mathrm{ZC}$ sequences of $\operatorname{root} u$ with shifts $C_{\mu}$ and $C_{v}=$ $\left(C_{\mu}+\tau \frac{M_{a}}{M}\right) \bmod N_{\mathrm{ZC}}$, respectively. The function that allows us to gain insight into the effect of the TO is given by

$$
\frac{N}{\left|N_{\mathrm{ZC}}\right|^{2}}\left|\sum_{m=0}^{\frac{M_{a}}{2}-1-\tau \frac{M_{a}}{M}} e^{-j \frac{2 \pi}{N_{\mathrm{ZC}}} u\left(C_{\mu}+\tau \frac{M_{a}}{M}\right) m} e^{j \frac{2 \pi}{N_{\mathrm{ZC}}} u C_{v} m}\right|^{2} .
$$

It is worth emphasizing that the magnitude of the peaks decreases as $\tau$ increases. To avoid that a time-shifted version of the original sequence causes a peak above the noise floor, the shifts must be carefully selected as

$$
C_{v}=v N_{\mathrm{CS}}, \quad v=0, \cdots,\left\lfloor\frac{N_{\mathrm{ZC}}}{N_{\mathrm{CS}}}\right\rfloor-1 .
$$

The same criterion has been adopted in [7] to define the random access preambles for unrestricted preamble sets. The spacing between candidate sequences is controlled by $N_{\mathrm{CS}}$, which is judiciously selected to accommodate all possible time uncertainties.

Now we assume for simplification that the TO is negligible, i.e., $\tau=0$. In such a case, the $k$-th block approximately reduces to $\mathbf{D}_{\epsilon} \mathbf{x}[k]$. Analogously to SC-FDM [9], the effect of the CFO translates into a pure cyclic shift. In this circumstances, the correlation peak position is shifted. To compute the shift we shall identify the candidate sequences that maximize the 
TABLE I

SYSTEM PARAMETERS

\begin{tabular}{|l|l|l|l|}
\hline & SC-FDM M & SC-FDM ZC & FBMC \\
\hline Multicarrier symbols & $N=1$ & $N=1$ & $N=2$ \\
\hline Subcarriers & $M=1536$ & $M=1536$ & $M=1536$ \\
\hline Active subcarriers & $M_{a}=1023$ & $M_{a}=839$ & $M_{a}=840$ \\
\hline Subcarrier spacing & $\Delta_{f}=1.25 \mathrm{KHz}$ & $\Delta_{f}=1.25 \mathrm{KHz}$ & $\Delta_{f}=1.25 \mathrm{KHz}$ \\
\hline Frequency sampling & $f_{s}=1.92 \mathrm{MHz}$ & $f_{s}=1.92 \mathrm{MHz}$ & $f_{s}=1.92 \mathrm{MHz}$ \\
\hline PRACH bandwidth & $B_{\mathrm{PRACH}}=1.31 \mathrm{MHz}$ & $B_{\mathrm{PRACH}}=1.08 \mathrm{MHz}$ & $B_{\mathrm{PRACH}}=1.08 \mathrm{MHz}$ \\
\hline Transmit antennas & $N_{\mathrm{tx}}=1$ & $N_{\mathrm{tx}}=1$ & $N_{\mathrm{tx}}=1$ \\
\hline Receive antennas & $N_{\mathrm{rx}}=2$ & $N_{\mathrm{rx}}=2$ & $N_{\mathrm{rx}}=2$ \\
\hline
\end{tabular}

correlation with the received signal when the channel is represented by $\mathbf{D}_{\epsilon}$. Upon evaluating

$$
\begin{gathered}
\frac{N}{\left|N_{\mathrm{ZC}}\right|^{2}}\left|\sum_{m=0}^{\frac{M_{a}}{2}-1} e^{-j \frac{2 \pi}{N_{\mathrm{ZC}}} u C_{\mu} m}\left[\mathbf{D}_{\epsilon}\right]_{m m} e^{j \frac{2 \pi}{N_{\mathrm{ZC}}} u C_{v} m}\right|^{2}= \\
\frac{N}{\left|N_{\mathrm{ZC}}\right|^{2}}\left|\frac{\sin \left(\frac{\pi}{N_{\mathrm{ZC}}}\left(\epsilon \frac{N_{\mathrm{ZC}}}{M_{a}}-u C_{v}+u C_{\mu}\right) \frac{M_{a}}{2}\right)}{\sin \left(\frac{\pi}{N_{\mathrm{ZC}}}\left(\epsilon \frac{N_{\mathrm{ZC}}}{M_{a}}-u C_{v}+u C_{\mu}\right)\right)}\right|^{2}
\end{gathered}
$$

we can determine if the shift $C_{v}$ could be mistaken for the real shift $C_{\mu}$. In the SC-FDM scheme evaluated in [9] the correlation between two sequences with a CFO $\frac{2 \pi}{N_{\mathrm{ZC}}} \epsilon$ is

$$
\frac{1}{\left|N_{\mathrm{ZC}}\right|^{2}}\left|\frac{\sin \left(\pi\left(\epsilon-u C_{v}+u C_{\mu}\right)\right)}{\sin \left(\frac{\pi}{N_{\mathrm{ZC}}}\left(\epsilon-u C_{v}+u C_{\mu}\right)\right)}\right|^{2} .
$$

In order to shred some light on the comparison between pruned DFT-s-FBMC with NCPDI and SC-FDM with conventional detection based on full-length correlation, we have depicted in Figure 5 the magnitude of the correlation as function of $\epsilon$. As for the parameters, we have set $N_{\mathrm{ZC}}=839$, $M_{a}=840$ and $N=2$. In the example illustrated in Figure 5 , the transmitted preamble is an arbitrary sequence that is characterized by a root index $u$ and a cyclic shift $C_{\mu}$. The sequences that are generated locally use the same root index, while the shifts are given by $C_{v}=C_{\mu}, C_{v}=C_{\mu} \pm d_{u 1}$ and $C_{v}=C_{\mu} \pm d_{u 2}$. The variable $d_{u n}$ is the cyclic shift that coincides with a CFO of magnitude $\frac{2 \pi}{N_{\mathrm{ZC}}} n$ and is computed by solving $n=u d_{u n} \bmod N_{\mathrm{ZC}}$. Analyzing the shape of the correlation, it becomes apparent that in SC-FDM the lobes are narrower and decay faster. The step decay of the correlation function indicates that the detector suffers from a performance degradation in presence of fractional $\epsilon$.

\section{NUMERICAL RESULTS}

This section evaluates the random access procedure in presence of large $\mathrm{CFO}$ values, which are assumed to produce a constant rotation during the preamble transmission. The system parameters are listed in Table I. While the baseline PRACH design described in [7] is identified with the acronym SC-FDM ZC, we use the term FBMC to refer to the proposed preamble design and detection method. As a benchmark we have also considered the solution conceived in [15], where the SC-FDM modulation format is used to carry $m$-sequences. This random access preamble design is referred to as SCFDM M. Concerning the detection method, both SC-FDM ZC and SC-FDM M, resort to full-length correlation. It should

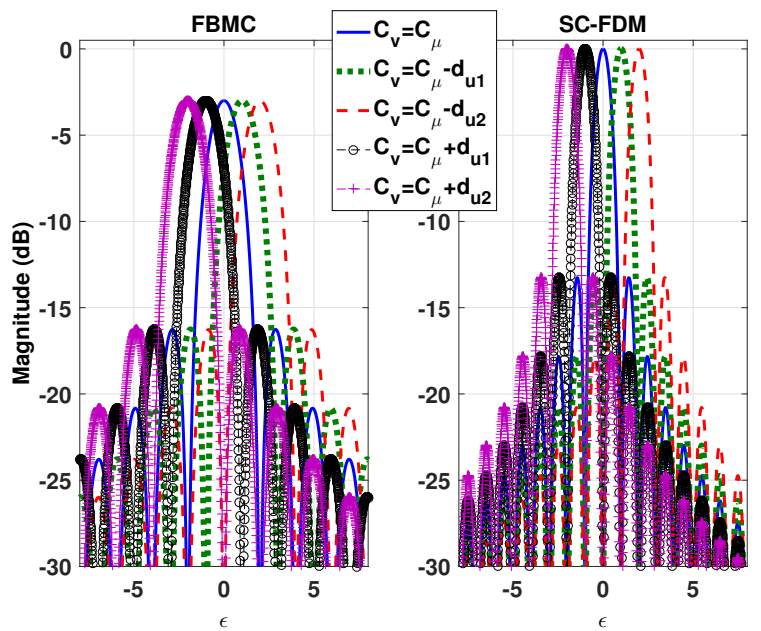

Fig. 5. Magnitude of the correlation in pruned DFT-s-FBMC with NCPDI and SC-FDM with detection based on full-length correlation as function of the normalized CFO.

be noted that the number of active carriers in FBMC is an even number. This constraint is imposed by the preamble design addressed in Section IV, which fixes the number of data symbols to $M_{a} / 2$.

For the sake of fairness in the comparison, both SC-FDM $\mathrm{ZC}$ and FBMC follow the same procedure to generate the preamble sequences. That is, the root indices and the cyclic shifts are computed according to [7]. In this regard, the UEs are configured to use the restricted preamble set type B, which is created to establish uplink synchronization in high mobility scenarios. To show the potential of the proposed solution, the example considered is based on the PRACH test for burst format 1 [26]. Starting from the root index $u=7$, a preamble set of $64 \mathrm{ZC}$ sequences is formed. The most relevant parameters are gathered in Table II. In SC-FDM M, the guidelines reported in [15] have been followed to generate the preamble set.

Attention must be drawn to the fact that the FBMC configuration is slightly modified to ensure that the new random access signal does not last longer than the baseline PRACH. Essentially, the difference stems from the fact that the size of the sequence in samples is $\frac{M}{2}(N+1)$ and $M$ in FBMC and SC-FDM, respectively. According to the burst format 1 , the GT, the sequence and the CP/ZP block, must be allocated in two subframes $(2 \mathrm{~ms})$. It therefore follows that the duration of the ZP block and the GT must be reduced when FBMC is 
TABLE II

PRACH PARAMETERS FOR BURST FORMAT 1

\begin{tabular}{|l|l|l|l|}
\hline & SC-FDM M & SC-FDM ZC & FBMC \\
\hline Sequence duration & $T_{\mathrm{SEQ}}=800 \mu \mathrm{s}$ & $T_{\mathrm{SEQ}}=800 \mu \mathrm{s}$ & $T_{\mathrm{SEQ}}=1200 \mu \mathrm{s}$ \\
\hline GT duration & $T_{\mathrm{G}}=515.63 \mu \mathrm{s}$ & $T_{\mathrm{G}}=515.63 \mu \mathrm{s}$ & $T_{\mathrm{G}}=315.63 \mu \mathrm{s}$ \\
\hline CP/ZP duration & $T_{\mathrm{CP}}=684.37 \mu \mathrm{s}$ & $T_{\mathrm{CP}}=684.37 \mu \mathrm{s}$ & $T_{\mathrm{ZP}}=484.37 \mu \mathrm{s}$ \\
\hline Preamble length & $N_{\mathrm{M}}=1023$ & $N_{\mathrm{ZC}}=839$ & $N_{\mathrm{ZC}}=839$ \\
\hline Length of zero correlation zone & $N_{\mathrm{CS}}=100$ & $N_{\mathrm{CS}}=100$ & $N_{\mathrm{CS}}=100$ \\
\hline
\end{tabular}
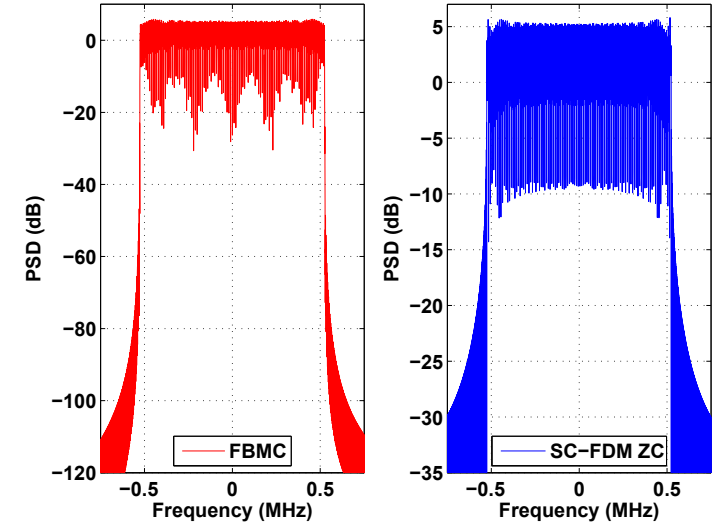

Fig. 6. Power spectral density of the transmitted signal in a $1.5 \mathrm{MHz}$ bandwidth.

the waveform of choice. This may suggest that the proposed preamble design increases the leakage to the next subframe and exhibits more vulnerability to the interference that comes from the previous subframe. However, it is important to remark that thanks to the pruned DFT precoding, the energy of the first and last $M / 4$ samples of the burst is significantly lower that the average power. It can be concluded that the immunity of the system to self-interference goes beyond the GT and the ZP block. Therefore, the effective length of the GT and the $\mathrm{ZP}$ block is increased in approximately $M / 4$ samples, which is equivalent to $200 \mu s$.

\section{A. Out-of-band emissions}

The FBMC scheme evaluated in this section uses the timefrequency localization prototype pulse [24]. Figure 6 depicts the power spectral density (PSD) in a $1.5 \mathrm{MHz}$ bandwidth. To obtain the plots, the random access preamble signals have been normalized to have unit energy. Predictably, the side lobes in FBMC have a step decay and thus, the interference caused by OOB emissions is reduced when compared to SC-FDM ZC. This allows the system to make the most efficient use of spectral resources, by using available spectral holes and reducing the subcarriers that remain silent at the edges of the band.

\section{B. Peak-to-average power ratio}

The performance of the PRACH also depends on the envelope fluctuation. To get insightful information, it is common to represent the cumulative distribution function (CDF) of the PAPR. Figure 7 shows that ZC sequences achieve lower PAPR

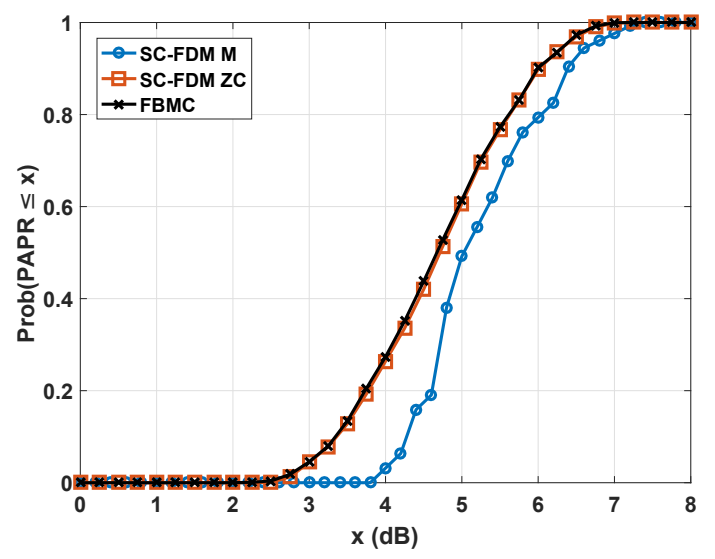

Fig. 7. CDF of the PAPR.

compared to $m$-sequences. That is because the application of the DFT matrix in the SC-FDM M scheme does not result in a constant modulus sequence. By contrast, in SC-FDM ZC, the DFT does not destroy the constant envelope property of $\mathrm{ZC}$ sequences. In addition, we show that FBMC performs as well as SC-FDM ZC, which is in alignment with the results presented in [17].

\section{Missed detection probability}

In this section the performance is evaluated in terms of missed detection probability. At each random access opportunity only a single user is active. The position of the user is uniformly distributed over the cell. According to the $N_{\mathrm{CS}}$ value proposed by the PRACH test in [26], the RTD shall not exceed $95.24 \mu \mathrm{s}$. Then, the detector is able to distinguish the peaks associated with different delays. For single-user access, the error cases that lead to an erroneous detection include the detection of different preamble than the one that was sent, not detecting a preamble at all or correct preamble detection but with the wrong timing estimation [26]. To comply with the performance requirements of the PRACH test defined in [26], a timing estimation error must be declared if the magnitude of the error is larger than $1.04 \mu$ s.

As for the propagation conditions, in satellite communication systems the multipath effect is usually weak while the LoS component is very strong. For this reason, the AWGN channel model has been adopted. It must be mentioned that the base station is equipped with two antennas to reduce the missed detection probability. The decision variables obtained by the two antennas are averaged, which allows using the detection threshold devised for single antenna architectures. 

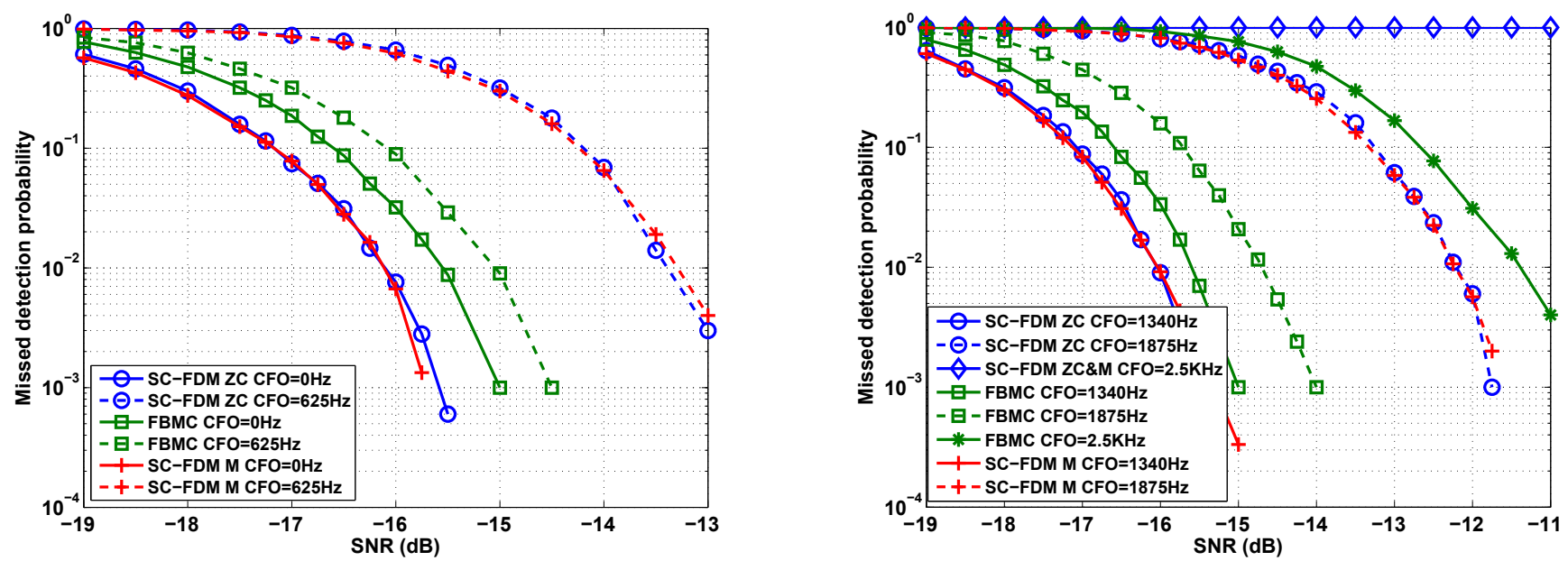

Fig. 8. Missed detection probability versus SNR in SC-FDM and FBMC for different values of CFO. For each preamble sequence, the detector performs 3 hypothesis tests.
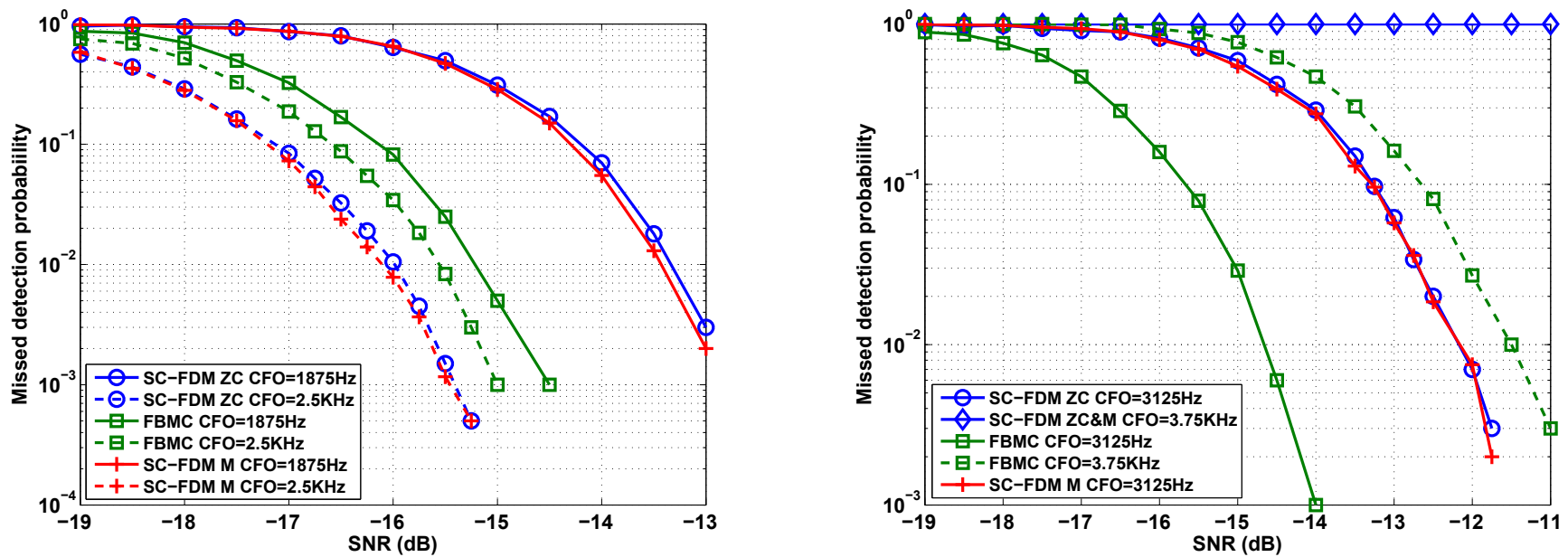

Fig. 9. Missed detection probability versus SNR in SC-FDM and FBMC for different values of CFO. For each preamble sequence, the detector performs 5 hypothesis tests.

In this regard, the detection threshold is computed assuming perfect knowledge of the noise power $N_{0}$, fixing the false alarm probability to $P_{\mathrm{FA}}=10^{-3}$ and setting the search window size to $D_{w}=N_{\mathrm{CS}} \frac{M}{M_{a}}$. Subject to these constraints, (37) is particularized for $N=2$ to obtain the threshold $r_{\text {th }}$ in FBMC. In the SC-FDM counterpart, regardless of the type of preamble sequence, we use $P_{\mathrm{FA}}=1-\left(1-e^{-\frac{r_{\mathrm{th}} N_{\mathrm{ZC}}}{N_{0}}}\right)^{D_{w}}$ to derive $r_{\mathrm{th}}$, [20].

It should be mentioned that the $\mathrm{CP}$ transmission allows the correlation window to capture all the relevant preamble samples, without the need of searching for the start of the sequence. This renders the use of sliding windows unnecessary, allowing a fast detection. To follow the same approach in the pruned DFT-s-FBMC context, the design of the CP block should be investigated to preserve the good frequency localization properties. All these aspects are deferred to future works. In anticipation that the proposed solution could be enhanced to reduce the complexity, the penalty for transmitting the CP will not be accounted in the SNR computation. This will give an idea of how the CP-based pruned DFT-s-FBMC would compare with SC-FDM.

It should be noted that SC-FDM $M$ requires a wider frequency range than do SC-FDM ZC and FBMC. Owing to this, the comparison may not be fair. To remedy this issue, a penalty factor of $10 \log _{10}\left(N_{\mathrm{ZC}} / N_{\mathrm{M}}\right) \mathrm{dB}$ has been introduced when SC-FDM M is evaluated.

Analyzing Figure 8 we can state that all schemes satisfy the performance requirements specified in [26]. To combat the CFO, two additional hypothesis tests are performed. That is, for each preamble of the set, two additional correlations are computed to compensate for a frequency offset of $\pm 1250 \mathrm{~Hz}$. From Figure 8 we can infer that the performance degradation is almost negligible when the $\mathrm{CFO}$ is close to $0 \mathrm{~Hz}$ or $\pm 1250 \mathrm{~Hz}$. In these positions the correlation function gets its maximum. It must be remarked that the missed detection probability is slightly higher in FBMC, for $\mathrm{CFO}=\{0, \pm 1340\}$ $\mathrm{Hz}$. By contrast, for fractional values of the subcarrier spacing, 


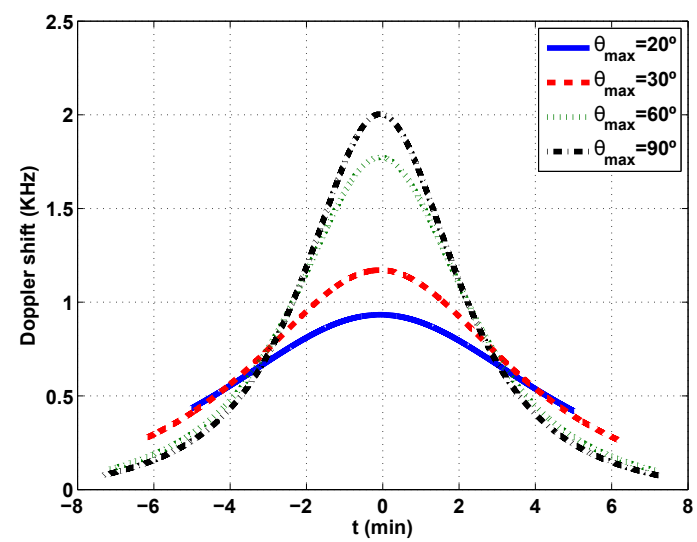

Fig. 10. Residual Doppler shift at the edge of a beam of radius $50 \mathrm{Km}$.

FBMC shows superior performance than the rest. Interestingly, FBMC is able to detect the preamble at $\mathrm{CFO}=2500 \mathrm{~Hz}$. As Figure 5 highlights, SC-FDM ZC cannot deal with such a frequency uncertainty, because the main lobe of the correlation is narrower.

Admittedly, SC-FDM ZC and SC-FDM M virtually deliver the same performance. The fundamental reason is that in both schemes the sequences are characterized by the constant amplitude property and the detection is based on the full-length correlation. However, it should be underlined that $m$-sequences are advantageous over ZC sequences in several aspects. For instance, the criterion to generate the preamble set is not so restrictive, which enable a higher number of sequences to be used within a cell [15].

To handle higher Doppler frequency shifts, detectors search for correlation peaks at the following frequency offset hypothesis: $0, \pm 1250 \mathrm{~Hz}$ and $\pm 2500 \mathrm{~Hz}$. Figure 9 corroborates that initial access could be granted in presence of large $\mathrm{CFO}$ values. In FBMC systems, up to $3750 \mathrm{~Hz}$ of frequency misalignment can be tolerated.

\section{Application scope}

The application scope of the proposed method is circumscribed to NGEO satellite systems that enable Doppler precompensation. Suppose that the Doppler frequency shift could be compensated on board as if the UE was at the beam center. Then, the maximum residual error will be observed at the edges of the beam. This case is illustrated in Figure 10. The maximum elevation angle ranges from $\theta_{\max }=20^{\circ}$ to $\theta_{\max }=90^{\circ}$. It is assumed that the orbit is inclined $87.9^{\circ}$, the altitude is $1200 \mathrm{~km}$, the carrier frequency is $2 \mathrm{GHz}$ and the beam radius is $50 \mathrm{~km}$ [27]. The highest frequency misalignment is at $t=0$, where the satellite is closest to the beam center. It is noteworthy that the uplink frequency offset is approximately two times the residual Doppler shift in the downlink. This observation highlights that the proposed method could tolerate the Doppler frequency uncertainty observed in Figure 10. To increase the beam size and keep the same robustness, it is required to use a larger subcarrier spacing.

\section{CONCLUSIONS AND FUTURE WORK}

In this paper, we introduce a new random access signal that achieves a high level of commonality with 5G NR. The novel scheme is based on modulating a ZC sequence onto a pruned DFT-s-FBMC waveform, without modifying the system parameters. The proposed solution outperforms in many aspects the standardized SC-FDM transmission with detection based on full-length correlation. It has much lower OOB emission and achieves a reduced missed detection probability in presence of high CFO. This has been showed analytically, by thoroughly analyzing the CFO impact on the algorithms for preamble detection, and by numerical simulations.

Potential applications of the proposed method include the initial access of 5G-based NGEO satellite networks, where frequency shifts are bound to arise. However, there are still some practical implementation aspects that need to be resolved. For instance, future work may consider developing more efficient algorithms for preamble detection. The transmission of redundancy in the form of a $\mathrm{CP}$ could reduce the complexity of the detector. However, inserting the $\mathrm{CP}$ into the structure of the pruned DFT-s-FBMC scheme may destroy its good frequency localization properties. Hence, it is worth exploring the most suitable structure, without modifying the features.

Another problem that should be addressed is the application of multiple-input multiple-output (MIMO) techniques to random access schemes. The multiplexing and diversity gains offered by the MIMO channel could be used to further increase the throughput yet these gains are questionable in satellite channels, as rich scattering is not encountered. To overcome this issue, polarized MIMO transmission may be employed. Nonetheless, further research is still needed to adapt the random access procedure to the MIMO setting.

Finally, an in-depth study would be also needed to determine if the advantages of $m$-sequences and the proposed scheme could be combined.

\section{APPENDiX A}

The Theorem 1 that is derived in [21] shows that the polyphase components of the prototype pulse are directly related to the perfect reconstruction property. In the particular case where $L=M$, the prototype pulse must satisfy

$$
p^{2}[l]+p^{2}\left[\frac{M}{2}+l\right]=c, \quad l=0, \cdots, M / 2-1,
$$

to guarantee a transmission in the absence of interference. In notation terms, $c$ is a constant. It is noteworthy to mention that in the literature we can find short pulse designs with $L=$ $M$ that fulfill (A.1), e.g. the time-frequency localization and the quadrature mirror prototype pulses, [24]. Bearing in mind (A.1), for $q=2,4, \cdots, M_{a}-2$, (9) can be recast as

$$
\begin{gathered}
\alpha_{q 0}[0]=\sum_{n=0}^{M / 2-1}\left(p^{2}[n]+p^{2}\left[n+\frac{M}{2}\right]\right) e^{-j \frac{2 \pi}{M} n q}= \\
c \sum_{n=0}^{M / 2-1} \cos \left(\frac{2 \pi}{M} n q\right)-j \sin \left(\frac{2 \pi}{M} n q\right)=0 .
\end{gathered}
$$

The last equality stems from the fact that the summation zone includes several periods of cosine and sine functions. It is 
important to remark that $\alpha_{00}[0]=1$, because amounts to the energy of the pulse. Finally, the perfect reconstruction property (10) highlights that $\alpha_{q 0}[0]$ must be real-valued for $q$ odd, since $\theta_{q}^{*}[k] \theta_{0}[k]$ is pure imaginary. Therefore, it can be concluded that the elements of $\alpha_{0}$ are either zero or real-valued, which verifies Proposition 1.

\section{APPENDIX B}

Focusing our attention on the case where $M_{a}=M$ and the vector $\alpha_{0}$ is real-valued, it follows easily that $\alpha_{q 0}[0]=$ $\alpha_{(-q) 0}[0]=\alpha_{\left(M_{a}-q\right) 0}[0]$ and thus, the eigenvalues formulated in (17) can be expressed as

$$
\begin{aligned}
\lambda_{m}^{0} & =\alpha_{00}[0]+\alpha_{\left(M_{a} / 2\right) 0}[0](-1)^{m}+ \\
& \sum_{q=1}^{M_{a} / 2-1} 2 \alpha_{q 0}[0] \cos \left(\frac{2 \pi}{M_{a}} q m\right)
\end{aligned}
$$

As a consequence, it can be resolved that $\boldsymbol{\Lambda}_{0} \in \mathbb{R}^{M_{a} \times M_{a}}$. This observation allows us to state that Proposition 2 holds true.

\section{APPENDiX C}

It is important to remark that most of the energy of the prototype pulse is usually concentrated in this frequency range $\omega \in\left[-\frac{2 \pi}{M} \frac{2 \pi}{M}\right]$. As a result, in most of the cases the transmultiplexer response is such that $\left|\alpha_{10}[0]\right|>>\left|\alpha_{q 0}[0]\right|$, for $q>1$. In this case, if Proposition 2 remains true, it follows immediately that (B.1) can be approximated to

$$
\lambda_{m}^{0} \approx \alpha_{00}[0]+2 \alpha_{10}[0] \cos \left(2 \pi m / M_{a}\right) .
$$

Therefore, the $M_{a} / 2$ dominant eigenvalues of $\mathbf{G}^{c}[0]$ are given by $\left\{\lambda_{M_{a} / 4+1}^{0}, \cdots, \lambda_{3 M_{a} / 4}^{0}\right\}$, as long as $\alpha_{10}[0]<0$.

\section{APPENDIX D}

Assuming that $\mathbf{G}_{\tau \epsilon}[l]$ is equivalent to $\mathbf{G}_{\tau \epsilon}^{c}[l]$, it follows that

$$
\xi_{m}^{l}=\sum_{q=0}^{M_{a}-1} g_{q 0}^{\tau \epsilon}[l] e^{-j \frac{2 \pi}{M_{a}} q m} .
$$

We should underline that $\mathbf{G}_{\tau \epsilon}^{c}[l]$ is generated from (41), upon imposing $g_{(-q) 0}^{\tau \epsilon}[l]=g_{(M a-q) 0}^{\tau \epsilon}[l]$, for $q=1, \cdots, M_{a} / 2$. Then, the eigenvalues are given by

$$
\begin{aligned}
& \xi_{m}^{l}=\sum_{q=0}^{\frac{M_{a}}{2}-1} g_{q 0}^{\tau \epsilon}[l] e^{-j \frac{2 \pi}{M_{a}} q m}+\sum_{q=1}^{\frac{M_{a}}{2}} g_{(-q) 0}^{\tau \epsilon}[l] e^{j \frac{2 \pi}{M_{a}} q m}= \\
& \sum_{n} e^{j \frac{2 \pi}{M} n \epsilon} p[n] p\left[n-\tau+l \frac{M}{2}\right]\left(\sum_{q=0}^{\frac{M_{a}}{2}-1} e^{-j \frac{2 \pi}{M_{a}} q\left(\frac{M_{a}}{M} n+m\right)}\right. \\
& +\sum_{q=1}^{\frac{M_{a}}{2}} e^{\left.j \frac{2 \pi}{M_{a}} q\left(\frac{M_{a}}{M} n+m\right)\right)}=\sum_{n} e^{j \frac{2 \pi}{M} n \epsilon} p[n] p\left[n-\tau+l \frac{M}{2}\right] \\
& \times \frac{\sin \left(\frac{\pi}{2}\left(\frac{M_{a}}{M} n+m\right)\right)}{\sin \left(\frac{\pi}{M_{a}}\left(\frac{M_{a}}{M} n+m\right)\right)} \frac{2 \cos \left(\frac{\pi}{2}\left(\frac{M_{a}}{M} n+m\right)\right)}{e^{-j \frac{\pi}{M_{a}}\left(\frac{M_{a}}{M} n+m\right)}}
\end{aligned}
$$

We assume that by simply computing the dominant term of the summation, which corresponds to $n=M-m \frac{M}{M_{a}}$, we can get a sufficiently accurate expression, i.e,

$$
\begin{aligned}
\xi_{m}^{l} \approx & e^{-j \frac{2 \pi}{M_{a}} m \epsilon} e^{j 2 \pi \epsilon} M_{a} \times \\
& p\left[M-m \frac{M}{M_{a}}\right] p\left[M-m \frac{M}{M_{a}}-\tau+l \frac{M}{2}\right] .
\end{aligned}
$$

It is worth highlighting that if $M$ is a multiple of $M_{a}$, then the equality holds.

\section{ACKNOWLEDGMENT}

This work has received funding from the ministry of Science, Innovation and Universities under project TERESATEC2017-90093-C3-1-R (AEI/FEDER,UE) and under grant RTI2018-099841-B-I00 and from the Catalan Government (2017-SGR-1479).

\section{REFERENCES}

[1] "3rd Generation Partnership Project; Technical Specification Group Radio Access Network; Study on New Radio (NR) to support non terrestrial networks; (Release 15).” 3GPP TR 38.811.

[2] L. Siyang, Q. Fei, G. Zhen, Z. Yuan, and H. Yizhou, "LTE-satellite: Chinese proposal for satellite component of IMT-Advanced system," China Communications, vol. 10, no. 10, pp. 47-64, Oct 2013.

[3] C. Niephaus, M. Kretschmer, and G. Ghinea, "QoS Provisioning in Converged Satellite and Terrestrial Networks: A Survey of the Stateof-the-Art," IEEE Communications Surveys Tutorials, vol. 18, no. 4, pp. 2415-2441, Fourthquarter 2016.

[4] A. Guidotti, A. Vanelli-Coralli, M. Caus, J. Bas, G. Colavolpe, T. Foggi, S. Cioni, A. Modenini, and D. Tarchi, "Satellite-enabled LTE systems in LEO constellations," in IEEE International Conference on Communications Workshops (ICC Workshops), May 2017.

[5] A. Guidotti, A. Vanelli-Coralli, T. Foggi, G. Colavolpe, M. Caus, J. Bas, S. Cioni, and A. Modenini, "LTE-based satellite communications in LEO mega-constellations," International Journal of Satellite Communications and Networking, 2018.

[6] A. Guidotti, A. Vanelli-Coralli, M. Conti, S. Andrenacci, S. Chatzinotas, N. Maturo, B. Evans, A. Awoseyila, A. Ugolini, T. Foggi, L. Gaudio, N. Alagha, and S. Cioni, "Architectures and Key Technical Challenges for 5G Systems Incorporating Satellites," IEEE Transactions on Vehicular Technology, vol. 68, no. 3, pp. 2624-2639, March 2019.

[7] "3rd Generation Partnership Project; Technical Specification Group Radio Access Network; NR; Physical channels and modulation; (Release 15)." 3GPP TS 38.211.

[8] S. Sesia, I. Toufik, and M. Baker, LTE, The UMTS Long Term Evolution: From Theory to Practice. Wiley Publishing, 2009.

[9] M. Hua, M. Wang, W. Yang, X. You, F. Shu, J. Wang, W. Sheng, and Q. Chen, "Analysis of the Frequency Offset Effect on Random Access Signals," IEEE Transactions on Communications, vol. 61, no. 11, pp. 4728-4740, Nov 2013.

[10] M. Hua, M. Wang, K. W. Yang, and K. J. Zou, "Analysis of the Frequency Offset Effect on Zadoff-Chu Sequence Timing Performance," IEEE Transactions on Communications, vol. 62, no. 11, pp. 4024-4039, Nov 2014

[11] G. Wu, H. Yuan, W. Guo, S. Hu, and S. Li, "On design of physical random access channel in high-speed railway LTE systems," Chinese Science Bulletin, vol. 59, no. 35, pp. 5042-5050, Dec 2014

[12] T. Li, W. Wang, and T. Peng, "An improved preamble detection method for LTE PRACH in high-speed railway scenario," in 10th International Conference on Communications and Networking in China, Aug 2015.

[13] G. Cui, Y. He, P. Li, and W. Wang, "Enhanced Timing Advanced Estimation With Symmetric Zadoff-Chu Sequences for Satellite Systems," IEEE Communications Letters, vol. 19, no. 5, pp. 747-750, May 2015.

[14] P. Li, Y. He, G. Cui, W. Wang, and Y. Zhang, "A novel timing advanced estimation algorithm for eliminating frequency offset in satellite system," in IEEE 26th Annual International Symposium on Personal, Indoor, and Mobile Radio Communications (PIMRC), Aug 2015.

[15] G. Schreiber and M. Tavares, "5G New Radio Physical Random Access Preamble Design," in 2018 IEEE 5G World Forum (5GWF), July 2018 , pp. 215-220. 
[16] "Chairman's notes of study on solutions for NR to support Non Terrestrial Network." 3GPP RAN1\#98 and RAN1\#98b.

[17] R. Nissel and M. Rupp, "Pruned DFT Spread FBMC: Low PAPR, Low Latency, High Spectral Efficiency," IEEE Transactions on Communications, vol. 66, no. 10, pp. 4811-4825, Oct 2018.

[18] A. J. Viterbi, CDMA: principles of spread spectrum communication. Redwood City, CA, USA: Addison Wesley Longman Publishing Co. Inc., 1995.

[19] R. Pedone, M. Villanti, A. Vanelli-Coralli, G. E. Corazza, and P. T. Mathiopoulos, "Frame synchronization in frequency uncertainty," IEEE Transactions on Communications, vol. 58, no. 4, pp. 1235-1246, April 2010.

[20] C. Rohde, N. Alagha, R. De Gaudenzi, H. Stadali, and G. Mocker, "Super-framing: a powerful physical layer frame structure for next generation satellite broadband systems," International Journal of Satellite Communications and Networking, vol. 34, no. 3, pp. 413-438, 2016.

[21] P. Siohan, C. Siclet, and N. Lacaille, "Analysis and design of OFDM/OQAM systems based on filterbank theory," IEEE Transactions on Signal Processing, vol. 50, no. 5, pp. 1170 -1183, may 2002.

[22] A. I. Perez-Neira, M. Caus, R. Zakaria, D. L. Ruyet, E. Kofidis, M. Haardt, X. Mestre, and Y. Cheng, "MIMO Signal Processing in Offset-QAM Based Filter Bank Multicarrier Systems," IEEE Transactions on Signal Processing, vol. 64, no. 21, pp. 5733-5762, Nov 2016.

[23] G. Berardinelli, F. M. L. Tavares, T. B. Sørensen, P. Mogensen, and K. Pajukoski, "Zero-tail DFT-spread-OFDM signals," in IEEE Globecom Workshops, Dec 2013

[24] J. Nadal, C. A. Nour, and A. Baghdadi, "Design and Evaluation of a Novel Short Prototype Filter for FBMC/OQAM Modulation," IEEE Access, vol. 6, pp. 19610-19625, 2018.

[25] L. Zhen, H. Qin, B. Song, R. Ding, X. Du, and M. Guizani, "Random Access Preamble Design and Detection for Mobile Satellite Communication Systems," IEEE Journal on Selected Areas in Communications, vol. 36, no. 2, pp. 280-291, Feb 2018.

[26] "3rd Generation Partnership Project; Technical Specification Group Radio Access Network; Evolved Universal Terrestrial Radio Access (EUTRA); Base Station (BS) radio transmission and reception (Release 15)." 3GPP TS 36.104

[27] I. Ali, N. Al-Dhahir, and J. E. Hershey, "Doppler characterization for LEO satellites," IEEE Transactions on Communications, vol. 46, no. 3 , pp. 309-313, March 1998.

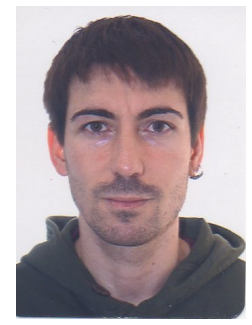

Màrius Caus received the $\mathrm{M} . \mathrm{Sc}$. and $\mathrm{PhD}$ (cum laude) degrees in Telecommunications engineering from the Universitat Politècnica de Catalunya (UPC), Barcelona, Spain, in July 2008 and December 2013, respectively. In 2009 he was granted by the Spanish Government to carry out his $\mathrm{PhD}$ studies in the Signal Theory and Communications Department at UPC. In 2018 he received the 2-year postdoctoral Juan de la Cierva fellowship from the Spanish Government. He has participated in several projects funded by the European Commission, the European Space Agency and the Spanish Ministry of Science. He is currently a Researcher at Centre Tecnològic de Telecomunicacions de Catalunya (CTTC), Castelldefels, Spain. His main research interests include filter bank based multicarrier systems, signal processing for communications and satellite communications.

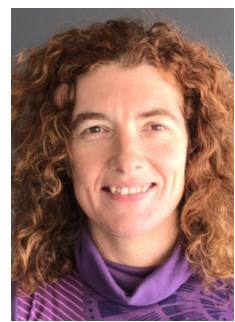

Ana I. Pérez-Neira (SM'02, FM'20) is full professor at Universitat Politècnica de Catalunya in the Signal Theory and Communication department since 2006 and was Vice rector for Research (2010-14). She is fellow researcher at Centre Tecnològic de Telecomunicacions de Catalunya, where she is the Coordinator of the SatCom and Space activities. Her research is in signal processing for communications, focused on satellite communications. She has more than 60 journal papers and 300 conference papers. She is co-author of 7 books. She has leaded more than 20 projects and holds 8 patents. She is the coordinator of the Network of Excellence on satellite communications, financed by the European Space Agency: SatnexIV. She has been associate editor of the IEEE TSP and EURASIP SP and ASP. Currently she is senior area editor of OJSP. She is member of the BoG of the IEEE SPS. She is IEEE Fellow and member of the Real Academy of Science and Arts of Barcelona (RACAB). She is recipient for the 2018 EURASIP Society Award and she is the general chair of IEEE ICASSP'20.

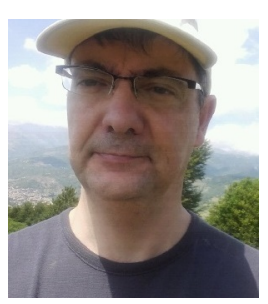

Joan Bas received the M.Sc and $\mathrm{PhD}$ (cum laude) in Electrical Engineering from the Universitat Politècnica de Catalunya (UPC) in 1998 and 2004 respectively. During the pursuit of his $\mathrm{PhD}$, he was recipient of a 1998-2000 fellowship of the UPC's Communication Signal Processing Group, and a 2000-2004 PhD scholarship granted by the Spanish Ministry of Science and Technology. In March 2005 he joined the Centre Tecnològic de Telecomunicacions de Catalunya (CTTC) as a postdoctoral researcher. In 2008 he was granted with a Leornardo Torres Quevedo fellowship from the Spanish Government to join CTTC as a permanent staff. Currently he holds at CTTC the position of Senior Research Associate in the Array \& Multi-Sensor department. His main areas of interest are: the improvement of the spectral and energy efficiency of satellite communications, the access contention in Machine-to-Machine (M2M) radio links and the security on IoT over Non Terrestrial Networks in licensed and unlicensed IoT systems.

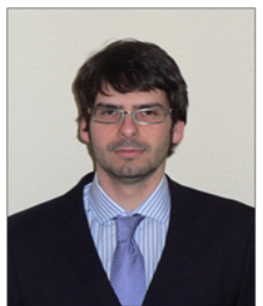

Luis Blanco received his MSc and $\mathrm{PhD}$ in Telecommunications engineering from the Polytechnic University of Catalonia (UPC), Spain, in 2006 and 2017, respectively. He holds a MSc degree in Research on Information and Communications Technologies (MERIT) from the UPC since 2012. Furthermore, he also has a degree in Data Science and Big Data from the University of Barcelona (UB) and a postgraduate degree in quantitative techniques for financial markets from the UPC, obtained in 2016 and 2012, respectively. Luis was recipient of the INNOVATIA award from IDE-CESEM (Instituto de Directivos de Empresa, Madrid). In 2007, he joined the Centre Tecnològic de Telecomunicacions de Catalunya (CTTC), where he currently holds a position as a researcher. He has actively participated in more than twenty research projects funded by public and private entities, including EC-funded projects (MONB5G, SEMANTIC, EMPhAtic, BeFEMTO, COOPCOM), national projects (e.g., 5G-TRIDENT, FBMC-SILAN, GRE3N), networks of excellence in research (SATNEX IV) with the European Space Agency (ESA) and a plethora of industrial projects with international companies (e.g., Huawei, Keysight technologies, Hispasat, etc.). His current research interests include machine learning for big data in 5G and beyond wireless systems, M2M/IoT over satellite networks, massive M2M communications for Internet of Things and dense wireless networks. 